\title{
Green Video Streaming in LTE Networks: A Client-Side Method for Power Efficiency of Mobile Devices
}

\author{
Jing-Yu Zhang, Yuan-Yi Chen, Xiao-Dong Meng, Chen-Tao Wu, Li-Ting Xu, Riaz Ali \\ Department of Computer Science and Engineering, Shanghai Jiao Tong University, Shanghai, China \\ E-mail: zhangzhang@sjtu.edu.cn, cyyxz@sjtu.edu.cn, xiaodongmeng1985@sjtu.edu.cn,wuct@ cs.sjtu.edu.cn, \\ xu-lt@ cs.sjtu.edu.cn, riaz_awkum@yahoo.com
}

\begin{abstract}
Nowadays mobile data traffic is soaring gradually year by year, the total monthly mobile data traffic (includes uplink and downlink) has come to more than 3,500 Petabytes and the growing trend is continuing. Video streaming demand is the significant contributor of cellular network data traffic, it accounted for more than $45 \%$ of mobile data traffic, and the occupancy percentage also is increasing. However the online video playing on mobile devices is still limited by battery technology which does not keep up with the development of cellular networks and mobile processor, mobile users may always need to charge the battery avoiding from device shutting down without power. Currently researchers focus on how to decrease mobile power consumption to prolong the battery lifetime and free mobile devices from charging cables. So it is urgent to understand and decrease the mobile power consumption of online video streaming in LTE networks. In this paper, we conduct a series of experiments to make a deep study of video streaming energy performance characteristics. We design some scenarios for collecting useful mobile power performance data and also measure the state-of-the-art video streaming performance and power consumption. We propose a client-side method to save the power consumption of mobile devices while the video streaming service is using. This method allows multiple video segments can be appended to the video player buffer each time. According to our experimental statistics, the mobile device can save up to more than $30 \%$ of the total power consumption for network part by using our multi-appending scheme for online video streaming.
\end{abstract}

Keywords-video streaming; LTE networks; mobile power consumption

\section{INTRODUCTION}

In recent years, the mobile industry is growing rapidly, and it seemingly is continuing to scale without any sign of faltering. The world is seeing a rapid technology migration to both higher speed mobile broadband networks and the increased adoption of smartphones and other connected devices. According to 2015 mobile economy statistics from the GSM Association (GSMA, or Groupe Speciale Mobile Association), in 2014 the mobile industry generated 3.8\% of global gross domestic product (GDP), a contribution that amounts to over US \$3 trillion of economic value across 236 countries [1].

Mobile industry development could always arouse a lot of attention not only in economic and social development area but also in research field recently. The accelerating technology shift to mobile broadband networks has been applied across the globe. A GSMA report shows it seems like 4G LTE networks should become dominant in the near future, the build out of LTE networks continued apace in 2014, with 335 networks having been deployed in 118 countries. It is predicted, the coverage of $4 \mathrm{G}$ LTE networks will reach half of the global population, and the total progress just only takes eight years.

On the other hand, the global monthly mobile traffic (includes uplink and downlink) in Q1 2015 has come to around 3,500 Petabytes and there is no evidence to prove this trend will stop [2]. If we look into some details of mobile data traffic growth, we can find out that mobile video traffic dominates the total traffic growth. In 2014, video accounted for around $45 \%$ of mobile data traffic. Mobile video in general is forecast to grow by around 55 percent annually through to 2020, when it will account for around 60 percent of all mobile data traffic.

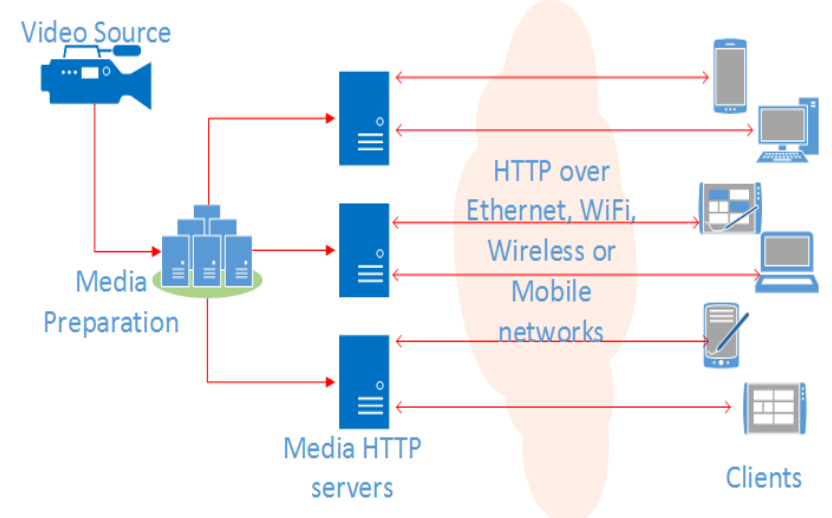

Figure 1. The typical architecture of a video streaming network.

Video streaming is the most popular technology used for online video playing back. In a typical video streaming implementation, the video/audio source is cut into many short segments ("chunks") and encoded to the desired delivery format. Chunks are typically 2 to 4 seconds long. The encoded chunks are hosted on an HTTP Web server. A client requests the chunks from the Web server in a linear fashion and downloads them using plain HTTP progressive download. As the chunks are downloaded to the client, the client plays back the sequence of chunks in linear order. Because the chunks are carefully encoded without any gaps or overlaps between them, the chunks play back as a seamless video. Figure 1 shows a typical video streaming network. 


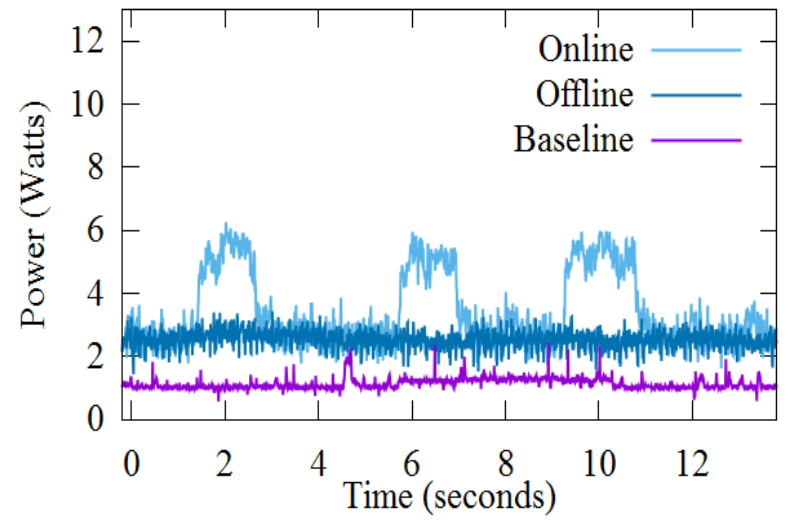

Figure 2. The distribution of power consumption. The Baseline, Offline and Online curves denote the power consumption when no video is played back on our mobile phone, when the mobile phone plays back the video offline, and when the mobile phone plays back the video online in 4G LTE networks, respectively.

Video streaming has been attracting increasing attention over the years, and it should play a significant role in mobile ecosystem in the near future. There are a lot of related research directions: (1) evaluation/analysis of power consumption in wireless networks [3-5]; (2) power consumption related to video streaming [6-8]; and (3) power consumption in 4G LTE Network [9-11]. However, most of the related work did not cover the mobile power consumption of online video streaming in LTE networks, which motivates our work in this paper.

In this work, we focus on video streaming in 4G LTE networks. 4G LTE improved the network performance pretty much comparing with some previous technologies. Video streaming also has been the mainstream for online video playing. So video streaming in 4G LTE networks should be one of the hottest research topics now and in the future. Figure 2 depicts the distribution of power consumption (obtained from our experiments), from which we can get a preliminary intuition on the power consumption of video streaming. In this paper we summarize our major contributions as follow.

- We design and implement a series of specific experiments for video streaming power and performance experiments, and we deploy a real mobile power monitoring system which the experiments are running on. Our real mobile power monitoring system includes one online video server, client video player deployment, a suite of analysis programs and some measurement tools. This real mobile monitoring system can feed back the real power consumption information instead of an emulation/estimated value for later data analysis.

- To understand their relationship from a theoretical viewpoint, we formulate the power models, which characterize various scenarios and provide us some insights. The models could be of independent interest, and could be used to understand other features related to the power consumption and video streaming.
- Based on our understanding and findings from the power models, we propose a client-side method to save the power consumption of mobile devices while the video streaming service is using. This method allows multiple video segments can be appended to the video player buffer each time. According to our statistics, the mobile device can save up to more than $30 \%$ of the total power consumption for network part by using our multi-appending method for video streaming.

In the subsequent section, we introduce the preliminaries related to video streaming technology and 4G LTE network characteristics. Section III presents our mathematical models for online video streaming. We describe our systematic platform and the experimental results of our proposed method in Section IV. Section V concludes the paper with the interesting research topic.

\section{BACKGROUND}

In this section we cover necessary background knowledge of basic online video streaming theory and power-related LTE RRC state transitions. For each part we also should introduce some details relevant with mobile power consumption.

\section{A. Online Video Streaming}

The mainstream video online playing mechanism is video streaming, rather than progressive download, video streaming will not download a simple video file, but download a series of video small segments from an HTTP video server to local storage and playback them in sequence. The most common implementation of video streaming is HTTP-based online video streaming. In a typical online video streaming implementation, the video file from the video source will be split into many short segments (or chunks) and encoded to the desired delivery format [12]. Chunks are typically 2 to 4 seconds long. At the video codec level, this typically means that each chunk is cut along video GOP (Group of Pictures) boundaries (each chunk starts with a key frame) and has no dependencies on past or future chunks/GOPs.

As shown in Figure 1, it is a typical video streaming network. The captured video source will be split up into a series of file chunks with different resolutions by video service providers. And then the encoded chunks are hosted on HTTP web servers. A client uses an end device to request the chunks from a web server in a linear fashion and downloads them using plain HTTP progressive download. It uses a Media Representation Description (MPD) file to describe the video chunks information such as accessible segments and corresponding timing. Due to the video chunks are carefully encoded without any gaps and overlaps between them, the video chunks can play back as a seamless video. Today, many commercial companies has developed their own implementation technologies of adaptive video streaming, such as the proprietary Adobe HTTP Dynamic Streaming (HDS), Apple HTTP Live Streaming (HLS), Microsoft Smooth Streaming (MSS), and the only international standardized solution MPEG Dynamic 
Adaptive Streaming over HTTP (MPEG-DASH) follow nearly the same principle.

\section{B. LTE RRC State}

The Radio Resource Control (RRC) protocol belongs to the Universal Mobile Telecommunication System. The major functions of the RRC protocol include connection establishment and release functions, broadcast of system information, radio bearer establishment, reconfiguration and release, RRC connection mobility procedures, and paging notification [13]. In 4G LTE networks, there are only two main RRC states: RRC idle and RRC connected [15]. If there is no mobile data go through LTE networks, the RRC state is in idle mode. UE (such as the smartphone) should make the RRC state transition from RRC idle mode to RRC connected mode before transferring any application data or completing any signaling procedures [14].

Then we introduce the power consumption of RRC states in LTE networks. UE RRC state should be initialized as RRC idle at the beginning. When in this state, UE will be set in DRX (Discontinuous Reception) mode which will reduce the UE consuming power into a very low level. And after UE is trying to send/receive one packet, no matter how much the size is, the RRC state should be triggered into RRC connected state and corresponding UE power state should be promoted into a high level.

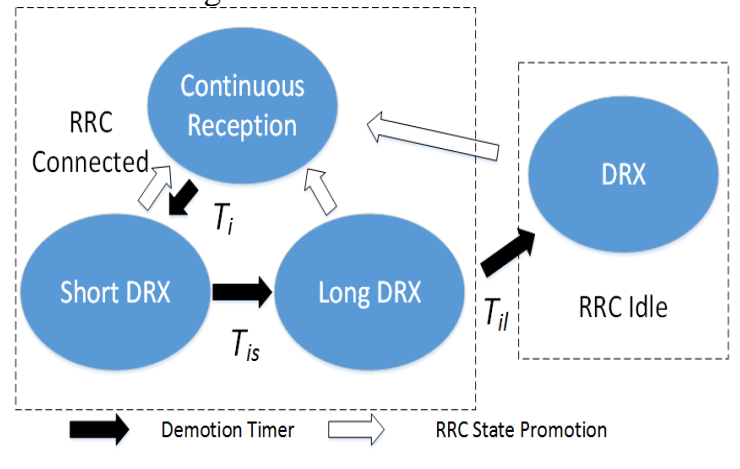

Figure 3. RRC state transitions.

Exactly, RRC connected state has three different submodes: 1) Continuous Reception, 2) Short DRX and 3) Long DRX, they may result in different power consumption at UEside [16]. When data transmission happened in LTE networks while UE is in RRC idle state, UE will immediately switch to continuous reception mode and it allows subsequent signaling to use a Dedicated Control Channel (DCCH) rather than the Common Control Channel $(\mathrm{CCCH})$. Once UE goes into continuous reception mode, one inactivity timer $T_{\mathrm{i}}$ (RRC inactivity timer) will be started. After $T_{\mathrm{i}}$ get expired if there is no data activities, the UE will go to Short DRX mode first rather than go to DRX mode. Once in Short DRX mode, anther inactivity timer $T_{\text {is }}$ (Short DRX cycle inactivity timer) starts. During $T_{\text {is }}$ duration, UE will probe data activity periodically controlled by the Short DRX cycle $T_{\mathrm{ps}}$. If any data transmission activity has been detected, the RRC should be changed into Continuous Reception state and the expected promotion time is much faster than from DRX mode, else UE should enter Long DRX mode. Long DRX mode is very similar with short DRX mode, but has different parameter settings for long DRX inactivity timer $T_{\mathrm{i} 1}$ and the Long DRX cycle $T_{\mathrm{pl}}$. If $T_{\mathrm{i} 1}$ expires, the RRC state will change into RRC idle state eventually. The $\mathrm{DCCH}$ will be released, and a $\mathrm{CCCH}$ should be assigned to UE for data activity probing.

As shown in Figure 3, regardless of in which RRC mode, when data transmission activity comes, the RRC mode will be changed into Continuous Reception state instantly. But the expected state promotion time is different. In Short DRX mode the promotion may take less time, and DRX mode may take the most. The demotion procedure from Continuous Reception to DRX mode will take a long path, and it only can be finished step by step controlled by different inactivity timers. Once any data transmission activity comes at any step, the RRC mode will be promoted into Continuous Reception mode.

\section{MATHEMATICAL MODELS}

In this section, we present mathematical models for the mobile phone power consumption related to the online video streaming in 4G LTE networks.

\section{A. Timing Scheme}

Before we formulate the power models for online video streaming, we show the timing scheme first. Figure 4 illustrates an overview of the timing scheme. Some terminologies mentioned here will be used extensively when we make the quantitative analysis on the energy/power consumption. The first step of online video streaming is the buffer filling stage, in this stage clients request enough segments to fill the video buffer. Then it comes to the buffer feeding stage which requires desired segments one by one to keep the buffer filled. The timing scheme of buffer feeding stage involves two levels:

1) Video streaming traffic level: At this level, there is an interactive process between the mobile client and the server (see the upper part of Figure 4). The mobile client sends periodically the video segment request to the video server. Once the video server receives such a request, it sends back the requested segment to the mobile client. When the requested segment is transmitted successfully, the mobile client sends the ACK message to the video server, and then enters the idle period, in which no network traffic is produced.

2) RRC level: At this level (see the bottom of Figure 4), the RRC state is in the connected mode during the segment transmission. Note that, after finishing the segment transmission, even if no new data transmission happens, the mobile client (actually, referred to the UE, recall previous section) still stays at the RRC connected state within a time duration (before entering the RRC idle state mode). The state in such a time interval is referred to as the RRC tail. 


\section{B. Power Model}

Because the energy consumption of buffer filling stage is fixed, we denote by $E_{\mathrm{bf}}$ the total energy consumes in this stage. Let $E$ be the total energy consumption of a mobile phone for network activities. Let $S$ and $C$ be the average download speed and the file size (of a video segment, i.e., chunk), respectively. Denote by $T_{\mathrm{s}}, T_{\mathrm{i}}, T_{\mathrm{rt}}, T_{\mathrm{ri}}$ the total service time of a segment, the idle period with no traffic, the RRC tail time duration, the RRC idle state time duration (in each transmission cycle, c.f., Figure 4), respectively. Let $P_{T_{(\cdot)}}$ denote the power consumption of the mobile phone during $T_{(.)}$(e.g., $P_{T_{(\mathrm{ri})}}$ denotes the power consumption during the RRC idle state time duration $\left.T_{(r i)}\right)$, and $P_{\text {off }}$ be the power consumption when playing back the video offline. Denote by $T_{\text {sd }}$ the playing back time duration of a video segment, and let $n$ be the number of total video segments for an online video streaming transmission after buffer filling stage. (For ease of reference, some notations used frequently are summarized in Table I.)

Recall previous subsection, the timing scheme involves two levels: (1) the video streaming traffic level, and (2) the RRC level. This implies that we can formulate our energy model from two routines. Specifically, for (1), we can have the following "basic" energy model:
TABLE I. NOTATIONS

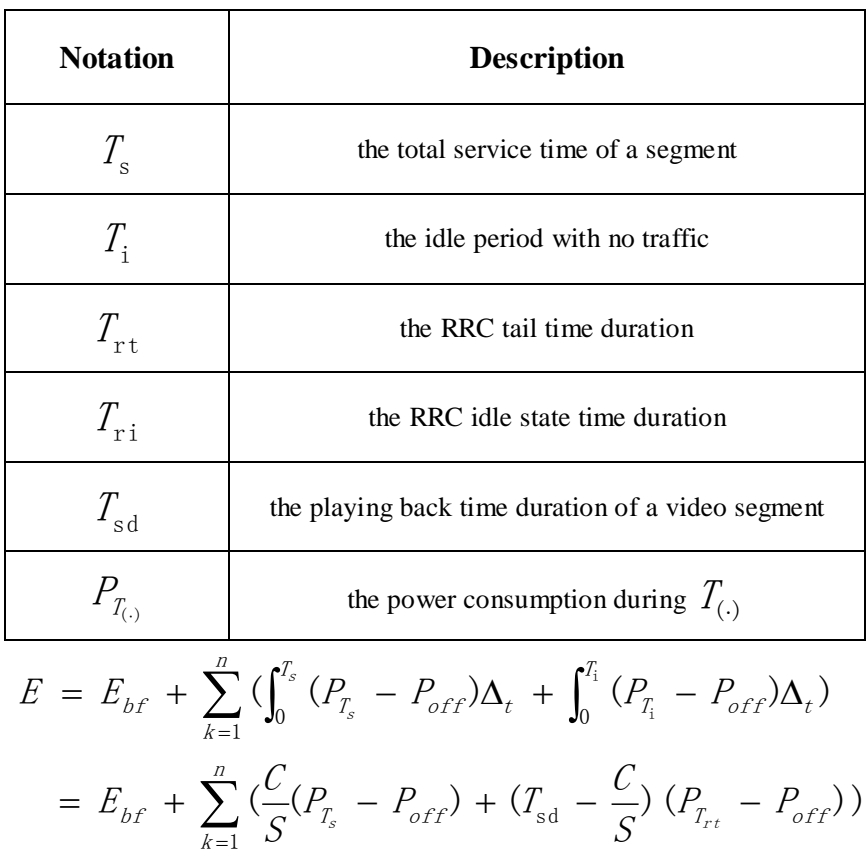

Observe that $T_{\mathrm{i}}$ equals the sum of $T_{\mathrm{rt}}$ and $T_{(r i)}$ (c.f., Figure 4). Thus, for (2), we have the "advanced" model (This model characterizes the "idle period with no traffic" in a more refined way) as follows.

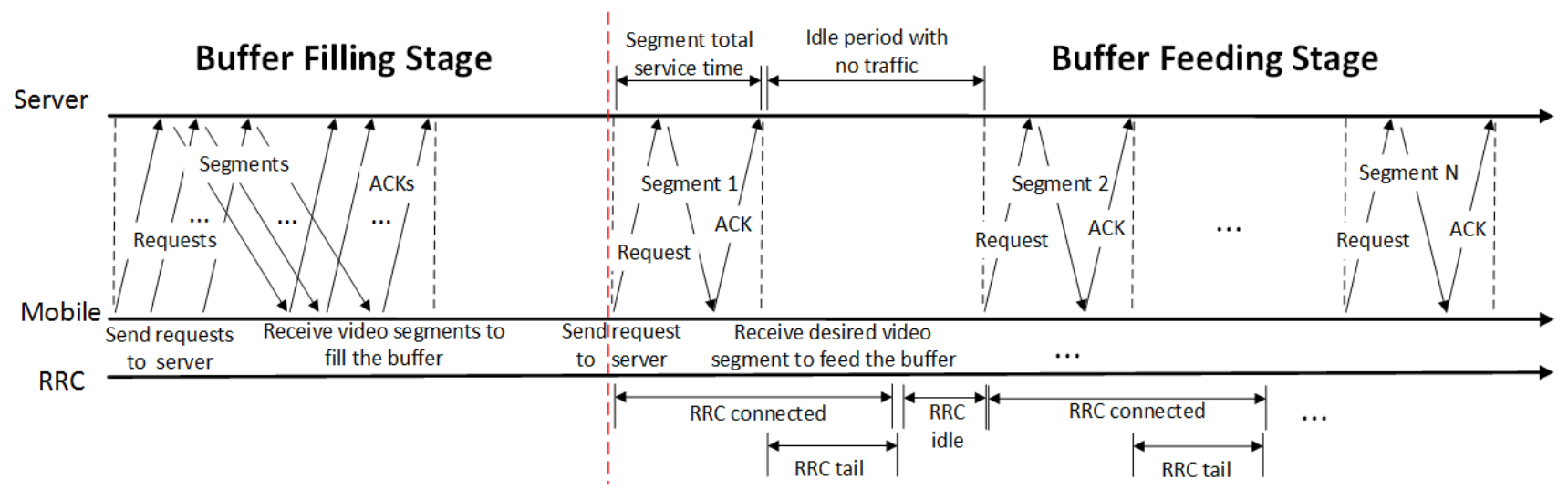

Figure 4. Example for the timing scheme.

$$
\begin{aligned}
E= & E_{b f}+\sum_{k=1}^{n}\left(\int_{0}^{T_{s}}\left(P_{T_{s}}-P_{o f f}\right) \Delta_{t}\right. \\
& \left.+\int_{0}^{T_{\mathrm{rt}}}\left(P_{T_{\mathrm{rt}}}-P_{o f f}\right) \Delta_{t}+\int_{0}^{T_{\mathrm{ri}}}\left(P_{T_{\mathrm{ri}}}-P_{o f f}\right) \Delta_{t}\right) \\
= & E_{b f}+\sum_{k=1}^{n}\left(\frac{C}{S}\left(P_{T_{s}}-P_{o f f}\right)+T_{\mathrm{rt}}\left(P_{T_{r t}}-P_{o f f}\right)\right. \\
& \left.+\left(T_{\mathrm{s} d}-\frac{C}{S}-T_{\mathrm{rt}}\right)\left(P_{T_{\mathrm{ri}}}-P_{o f f}\right)\right)
\end{aligned}
$$

\section{EXPERIMENTS}

\section{A. The Experimental Platform}

To investigate the power performance characteristics of video streaming in 4G LTE networks, we design a systematic platform. Our platform is composed of two major components: (1) hardware suite, which is responsible for video streaming system deployment and power data collection (see Figure 6); and (2) software suite, which is responsible for online video service, traffic data collection and analysis. The architecture of our platform is shown in 
Figure 5. In what follows, we introduce the details of our hardware and software suites.

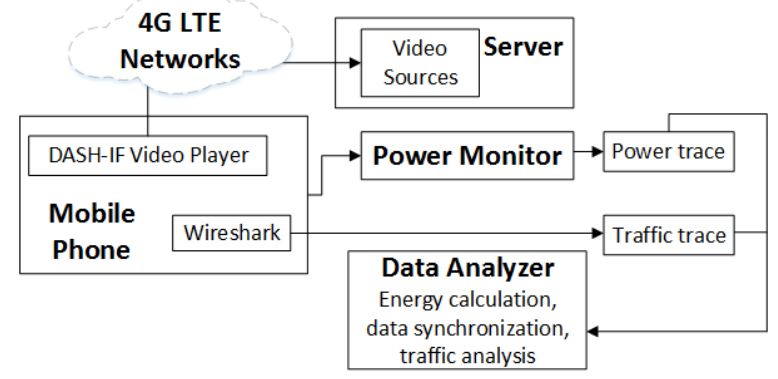

Figure 5. The architecutre of testing platform.

Our hardware suite mainly contains the following elements:

- We deploy a video web server that is connected with Internet using Ethernet cable, and is accessible via 4G LTE networks.

- We use an Android smart phone --- Samsung Galaxy S5 (one of the most popular smart phones) --- as our experimental mobile device. And we apply the $T$ Mobile 4G LTE high speed data plan (in United States) to the Android smart phone for our experiments.

- We use a professional mobile power meter --- the \{Monsoon power monitor\} (c.f., the white device in Figure 6) --- for mobile power data collection. Remark that many power measurement applications in "Android App market" may just use fixed values (pre-defined by smart phone manufacturers) to estimate the power consumption. Those power values could not reflect the exact real power values of smart phones. We here thus use the Monsoon power monitor to extract mobile power data.

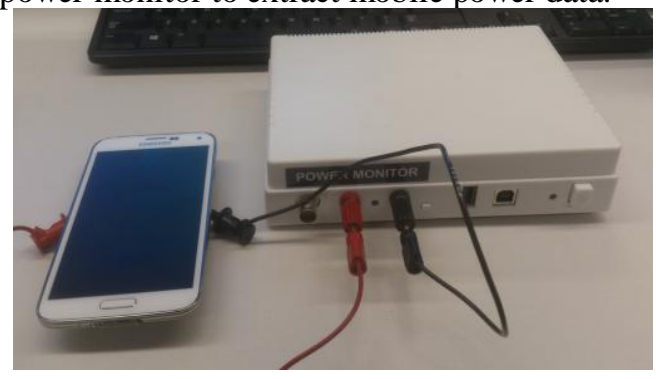

Figure 6. The testing devices.

Our software suite mainly includes a video player and some mobile data monitoring and diagnostic tools. Specifically,

- We deploy a video streaming player --- DASH-IF player (http://dashif.org/software) --- on the smart phone. (The DASH-IF player is the official DASH industry forum reference and production video streaming player.) The total length of the testing video is 10 minutes, and the segment length of our testing video is two seconds.

- Meanwhile, a widely used mobile data analyzer --Wireshark (https://www.wireshark.org) --- is built on our smart phone, in order to collect the traffic information in 4G LTE networks.

- The original operating system in our smart phone is Android 4.4.2 (Kitkat). We keep this system clean without other redundant applications, for the veracity of measurement.

- On the other hand, a servlet engine --- Jetty (http://www.eclipse.org/jetty) --- is deployed on the web server.

\section{B. Experimental Results and Analyses}

The conventional video segment appending scheme is to fetch the segments one by one periodically. In other words, each time the video player only fetches one segment to feed the buffer. We enable the multi-appending function in our platform. Denote by $M$ the multi-appending parameter. In the buffer feeding stage, each time $M$ video segment(s) will be appended to the video buffer using our multi-appending scheme. Note that, when $M=1$, the conventional singleappending scheme is using. We test with various resolutions (360p, 720p, and 1080p). The power values used in this work are the average values retrieved from our power monitor.

TABLE II. THE ABSOLUTE ONLINE POWER CONSUMPTION USING MULTI-APPENDING SCHEME

\begin{tabular}{|c|l|l|l|}
\hline $\begin{array}{c}\text { Multi-appending } \\
\text { Parameter }\end{array}$ & $\boldsymbol{M = 1}$ & $\boldsymbol{M = 2}$ & $\boldsymbol{M = 3}$ \\
\hline $\mathbf{3 6 0 p}$ & $335 \mathrm{~mW}$ & $267 \mathrm{~mW}$ & $235 \mathrm{~mW}$ \\
\hline $\mathbf{7 2 0 p}$ & $522 \mathrm{~mW}$ & $389 \mathrm{~mW}$ & $350 \mathrm{~mW}$ \\
\hline $\mathbf{1 0 8 0 p}$ & $620 \mathrm{~mW}$ & $469 \mathrm{~mW}$ & $429 \mathrm{~mW}$ \\
\hline
\end{tabular}

From Table II, we can notice that the absolute online power consumption (got by using the online power to minus the offline power) is decreasing when $M$ is getting larger. And the power improvemnt is ranging from $20 \%$ to $33 \%$ for different video resolutions.

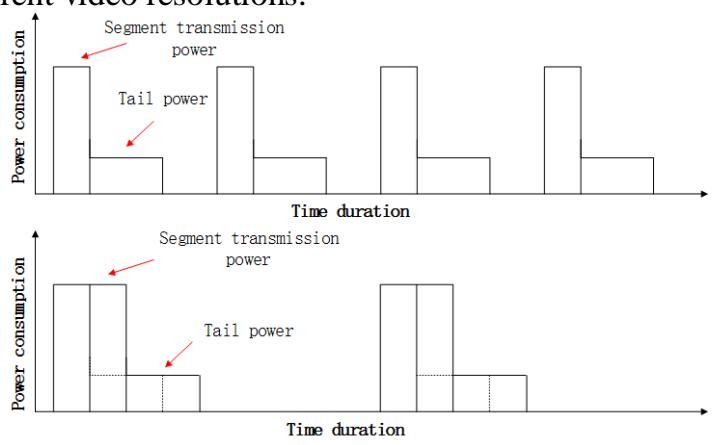

Figure 7. The comparison of different appending schemes.

According to Figure 7, we can understand the reason why the multi-appending scheme can save the power consumption. Because two video segments are appended to the video buffer together, the additional RRC tails which impact the total power consumption will be decreased. Another possible reason is that, the video segment transmission is usually finished before the TCP connection 
reaches a high speed if only one segment is being appended (recall that the TCP connection needs some time to warm up). This renders that the streaming pattern cannot achieve a high transmission speed when the single-appending scheme works, and multi-appending scheme can help the TCP connection gets a higher speed to shorten the transmission time. Less transmission time incurs less segment transmission power consumption.

\section{CONCLUSION}

We design and deploy our own measurement platform for online video streaming in LTE environment, and a series of experiments are conducted to take a deep study of online video streaming in $4 \mathrm{G}$ LTE networks. We then develop the analytical power models to identify and quantify the power inefficiency in mobile video streaming, due to the mismatch between HTTP request schedule and the radio resource control schedule. Based on the findings from our mathematical models, we propose a client-side method to save the power consumption of mobile devices while the video streaming service is using. This method allows multiple video segments can be appended to the video player buffer each time. As our experimental results shows, in LTE networks, the proposed scheme can save up to more than $30 \%$ of the total power consumption for network part for online video streaming.

\section{ACKNOWLEDGMENT}

This work was supported by the National Basic Research ("973") Program of China (2015CB352403), the National Natural Science Foundation of China (61261160502 and 61272099), the Scientific Innovation Act of STCSM (13511504200), and the EU FP7 CLIMBER project (PIRSES-GA-2012-318939).

\section{REFERENCES}

[1] GSMA mobile economy 2015. http://gsmamobileeconomy.com/global/

[2] Ericsson mobility report. http://www.ericsson.com/mobility-report, 2015.
[3] H. Wang, H. E. Roman, L. Yuan, Y. Huang, and R. Wang. Connectivity, coverage and power consumption in large-scale wireless sensor networks. Computer Networks, 75:212-225, 2014

[4] N. Zhu and A. V. Vasilakos. A generic framework for energy evaluation on wireless sensor networks. Wireless Networks, 22(4):1199-1220, 2016.

[5] L. Xiang, X. Ge, C.-X. Wang, F. Y. Li, and F. Reichert. Energy efficiency evaluation of cellular networks based on spatial distributions of traffic load and power consumption. IEEE Trans. on Wireless Communications, 12(3):961-973, 2013.

[6] Y. O. Sharrab and N. J. Sarhan. Aggregate power consumption modeling of live video streaming systems. In Proc. of the ACM International Conference on Multimedia System, pages 60-71, 2013.

[7] Y. Go, O. C. Kwon, and H. Song. An energy-efficient HTTP Adaptive Video Streaming with networking cost constraint over heterogeneous wireless networks. IEEE Trans. on Multimedia, 17(9):1646-1657, 2015.

[8] W. Hu and G. Cao. Energy-aware video streaming on smartphones. In Proc. of the International Conference on Computer Communications, pages 1185-1193, 2015.

[9] R. Imran, M. Shukair, N. Zorba, and C. Verikoukis. An energy saving strategy for LTE-A multiantenna systems. Mobile Networks and Applications, 20(5):692-700, 2015.

[10] M. Deruyck, E. Tanghe, D. Plets, L. Martens, and W. Joseph. Optimizing LTE wireless access networks towards power consumption and electromagnetic exposure of human beings. Computer Networks, 94:29-40, 2015.

[11] L.-P. Tung, Y.-D. Lin, Y.-H. Kuo, Y.-C. Lai, and K. M. Sivalingam. Reducing power consumption in LTE data scheduling with the constraints of channel condition and QoS. Computer Networks, 75:149-159, 2014.

[12] A. Zambelli. IIS smooth streaming technical overview. Microsoft Corporation, 3, 2009.

[13] European Telecommunications Standards Institute. UMTS RRC protocol specification (version 12.4.0 release 12), 2015.

[14] 3GPP tr 25.813: Radio interface protocol aspects (v7.1.0), 2006.

[15] Z. Zhang, Z. Zhao, H. Guan, D. Miao, and Z. Tan. Study of signaling overhead caused by keep-alive messages in lte network. In Proc. of IEEE 78th Vehicular Technology Conference (VTC Fall), pages 1-5. 2013.

[16] J. Huang, F. Qian, A. Gerber, Z. M. Mao, S. Sen, and O. Spatscheck. A close examination of performance and power characteristics of $4 \mathrm{G}$ LTE networks. In Proc. of the ACM 10th international conference on mobile systems, applications, and services, pages 225-238. 2012. 